\title{
STRICTLY ERGODIC MODELS FOR DYNAMICAL SYSTEMS
}

\author{
BY BENJAMIN WEISS
}

The action of a group $G$ by homeomorphisms of a compact metric space $X$ is said to be strictly ergodic if there is a unique Borel probability measure $\mu$ fixed by the action, and $\mu(U)>0$ for every nonempty open set $U \subset X$. For commutative groups $G$ (as well as for general amenable groups) this implies that the action is minimal, since if $X_{0} \varsubsetneqq X$ is closed and $G$-invariant there would exist a $G$-invariant measure supported by $X_{0}$ which would necessarily be different from $\mu$. Analogously one sees that the dynamical system $(X, G, \mu)$ must be ergodic. A remarkable result due to $\mathrm{R}$. Jewett [Je] and W. Kreiger $[\mathbf{K}]$ says that for $G=\mathbf{Z}$, any ergodic action is isomorphic to a strictly ergodic system. This was extended to $G=\mathbf{R}$ by K. Jacobs [Ja] and M. Denker and E. Eberlein [DE]. Thus the topological property of strict ergodicity places no restriction on the measure theoretic properties beyond the obvious ergodicity. It is natural to ask what happens for more general groups $G$, and what happens, even in the case of $\mathbf{Z}$, when we look at diagrams in the category of ergodic Z-actions rather than simply the objects themselves. In brief our results are:

(1) When $G$ is commutative every ergodic action has a strictly ergodic model.

(2) Any diagram in the category of ergodic Z-actions with the structure of an inverted tree, i.e., no portion of it looks like

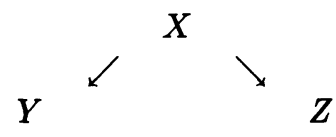

has a strictly ergodic model (as a diagram). However, not every measure theoretic triple

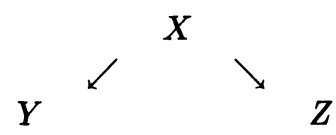

can have a strictly ergodic model.

As a consequence of (2), we can, for example, take any ergodic $\mathbf{Z}$-action that has some point spectrum and provide for it a strictly ergodic model in which all the eigenfunctions are continuous. One can combine (1) and (2) which was formulated for $\mathbf{Z}$-actions for those interested in the classical situation.

The method of proof that was developed for (1) is flexible enough to admit further refinements. For example, suppose that $G=\mathbf{Z}^{2}$, and the action is given by a pair of commuting transformations $T, S$ which are known to

Received by the editors December 7, 1984.

1980 Mathematics Subject Classification. Primary 28D05; Secondary 54H20. 
be separately ergodic. Then we can find a strictly ergodic model $(X ; \tau, \sigma)$ where separately $(X, \tau),(X, \sigma)$ are strictly ergodic as Z-actions. Looking to more general $G$ 's, the proof can be adapted to handle the so-called elementary amenable groups (cf. C. Chou $[\mathbf{C}]$ ), but it becomes necessary to assume, in addition to the ergodicity, the freeness of the action. ${ }^{1}$ This is not a consequence of strict ergodicity but rather comes from the fact that the proof relies heavily on the existence of Rohlin towers for which one needs the freeness of the action.

I would like to thank the MSRI for their hospitality during the 1983-1984 Dynamics year when this work was done.

1. $\mathbf{Z}^{2}$-actions. In what follows I will give a sketch of the proof of the following theorem, which is the easiest case of (1) above, and already contains the main elements of the more general results.

THEOREM 1. If $\left(Y, C, \nu ; T_{1}, T_{2}\right)$ is an ergodic $\mathrm{Z}^{2}$-action with generators $T_{1}, T_{2}$ then there exists a strictly ergodic $\mathbf{Z}^{2}$-system $\left(X, \tau_{1}, \tau_{2}\right)$ with unique invariant measure $\mu$, such that $\left(X, \tau_{1}, \tau_{2}, \mu\right)$ is measure theoretically isomorphic to $\left(Y, C, \nu ; T_{1}, T_{2}\right)$.

Let's call a set $C \in C$ uniform if

$$
\lim _{n \rightarrow \infty}\left\|\frac{1}{\left|S_{n}\right|} \sum_{(i, j) \in S_{n}} 1_{C}\left(T_{1}^{i} T_{2}^{j} x\right)-\nu(C)\right\|_{\infty}=0
$$

where $S_{n}$ is the square $\{(i, j): \max (|i|,|j|) \leq n\}$. Our goal is to construct an algebra of uniform sets that is $\left(T_{1}, T_{2}\right)$-invariant and generates $C$. Standard techniques will then give an explicit strictly ergodic model. An intermediate goal is to construct some such nontrivial algebra without worrying about generating $C$, and this is what we will do now. The main device will be a nested sequence of "uniform" Rohlin towers. if

Definitions. (1) $A$ set $B \in C$ is the base of an $(n, M, \delta)$-uniform $R$-tower

(i) $B \cap T_{1}^{i} T_{2}^{j} B=\varnothing$ all $(i, j) \in S_{n} \backslash\{(0,0)\}$,

(ii) for a.e. $y \in Y$,

$$
\frac{\left|\left\{(i, j) \in S_{M}: T_{1}^{i} T_{2}^{j} y \in B\right\}\right| \cdot\left|S_{n}\right|}{\left|S_{M}\right|}>1-\delta .
$$

(2) A sequence of uniform $R$-towers $\left(B_{i} ;\left(n_{i}, M_{i}, \delta_{i}\right)\right)$ will be said to be nested if for all $y \in B_{i}, y^{\prime} \in B_{i^{\prime}}, i<i^{\prime}$ if

$$
S_{n_{i}} y \cap S_{n_{\boldsymbol{i}^{\prime}}} y^{\prime} \neq \varnothing
$$

then $S_{n_{i}} y \subset S_{n_{i^{\prime}}} y^{\prime}$. (Here we begin writing $S_{n} y$ in lieu of $\left\{T_{1}^{i} T_{2}^{j} y ;(i, j) \in\right.$ $\left.S_{n}\right\}$.)

${ }^{1}$ NOTE ADDED IN PROOF. Alan Rosenthal and I have succeeded in extending the results described here to amenable groups in general, with the added assumption that the action be free. 
(3) Finally, we will call a sequence well-nested if the uniformities persist even after some sequence of modifications in which the internal tilings of $S_{n_{i}} y, y \in B_{i}$ by $S_{n_{j}}{ }^{\prime} s$ with $j<i$ are interchanged.

After constructing a sequence of well-nested uniform $R$-towers

$$
B_{i},\left(n_{i}, M_{i}, \delta_{i}\right)
$$

we proceed as follows. Given a partition $P$, and some $\varepsilon>0$ we will show how to construct a $\bar{P}$, such that the algebra

$$
\bigcup_{n=1}^{\infty} \bigvee_{(i, j) \in S_{n}} T_{1}^{i} T_{2}^{j} \bar{P}
$$

is uniform, and $d(P, \bar{P})<\varepsilon$. Applying a refinement of the ergodic theorem, we find some $i_{1}$ large enough so that most of the $S_{n_{i_{1}}}-P$-names across $B_{i_{1}}$ have a 1-block distribution very close to the global distribution of $P$. Change $P$ to $P_{1}$ so that all $S_{n_{i_{1}}}-P_{1}$-names across $B_{i_{1}}$ have good 1-block distributions. The fact that $B_{i_{1}}$ is $\left(n_{i_{1}}, M_{i_{1}}, \delta_{i_{1}}\right)$-uniform gives us our first uniformity for $P_{1}$. Next we apply a refinement of the ergodic theorem again to find an $i_{2}$, so that $S_{n_{i_{2}}}-P_{1}$-names across $B_{i_{2}}$ have a good "2-block" distribution. Changing $S_{n_{i_{2}}}-P_{1}$-names which aren't good involves a change in $B_{1}$. This is where the fact that the sequence is well nested comes in. This procedure is iterated to give the required $\bar{P}$, and our intermediate goal has been achieved. The best way to finish the proof of the theorem is to use the techniques of $\S 2$ below to catch more and more of $C$.

2. The relative $\mathbf{J}-\mathbf{K}$ theorem. The basic result here is the following

THEOREM 2. If $(X, \tau)$ is a strictly ergodic $\mathbf{Z}$-action, and $(Y, C, \nu, T)$ is an ergodic measure theoretic extension of $(X, \tau)$, then there exists a strictly ergodic extension of $(\hat{X}, \hat{\tau})$ of $(X, \tau)$ isomorphic to the pair $Y \rightarrow X$.

For the proof one first finds, by well-known techniques, an extension of $(X, \tau)$ that is zero-dimensional but measure theoretically isomorphic. Then one reduces the theorem to the following

THEOREM 3. If $P$ is a finite partition of $Y$ so that the algebra $A=$ $\bigcup_{n=1}^{\infty} \bigvee_{-n}^{n} T^{j} P$ is uniform and $\mathbf{Q}$ is any finite refinement of $P$ and $\varepsilon>0$ is given, then there exists a $\overline{\mathbf{Q}}$, a finite refinement of $P$ such that

(i) $\bigcup_{n=1}^{\infty} \bigvee_{-n}^{n} T^{j} \overline{\mathbf{Q}}$ is uniform,

(ii) $d(\mathbf{Q}, \overline{\mathbf{Q}})<\varepsilon$.

The key observation here is to let the fact that the algebra generated by $P$ is uniform help us out. We will describe the first step in the process of constructing $\overline{\mathbf{Q}}$. In the usual way, the ergodic theorem says that for $n$ large enough most $Q-n$-names have a good 1-block distribution. Form an $R$-tower of height $n$, with base $B \in A$, that fills most of $Y$. Most of the $P$-pure columns have some good $Q$-refinements. For these, modify $\mathbf{Q}$ to $\mathbf{Q}_{1}$ so that all the Q-refinements have good $q$-block distribution. Denote by $B_{1}$ the base of the tower consisting of these $P$-pure columns. Note that $B_{1} \in A$, and fills most 
of $Y$, so since $A$ is uniform, for some $N$, and a.e. $y$, the $P-N$-name of $y$ is almost all in the tower above $B_{1}$, and so for a.e. $y$ the $Q_{1}-N$-name has good 1-block distribution.

In the next step, we will look at $P \vee\left\{B_{1}, Y \backslash B_{1}\right\}$-pure columns and continue the process. At each stage the uniformity will come from the fact that the good distributions will be on a very large set in the uniform algebra $A$. It is more or less routine to push this idea through and prove Theorem 3.

The fact that not all diagrams of the type

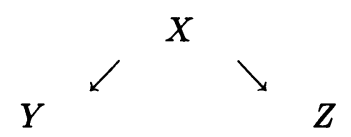

are realizable as strictly ergodic systems is a consequence of the following elementary result.

THEOREM 4. If $(X, \tau)$ is strictly ergodic with unique invariant measure $\lambda$ and $\pi:(X, \tau) \rightarrow(Y, \tau), \rho:(X, \tau) \rightarrow(Z, \tau)$ are factor maps with $\pi^{-1}(u) \cap$ $\rho^{-1}(v) \neq \varnothing$ for any nonempty open sets $U \subset Y, V \subset Z$ then $(Y, \tau, \pi \circ \lambda)$ and $(Z, \tau, \rho \circ \lambda)$ are measure theoretically disjoint.

It follows that if we take any weakly mixing process, say $(Y, T)$, and consider

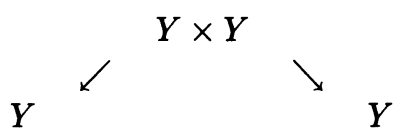

then this diagram cannot have a uniquely ergodic model, since naturally $(Y, T)$ is not measure theoretically disjoint from itself. It is not yet clear what the precise conditions on a diagram such as

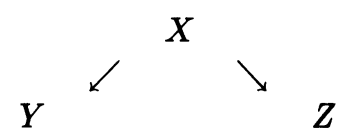

are that guarantee the existence of a strictly ergodic model.

\section{REFERENCES}

[C] Ching Chou, Elementary amenable groups, Illinois J. Math. 24 (1980), 396-407.

[DE] M. Denker and E. Eberlein, Ergodic flows are strictly ergodic, Adv. in Math. 13 (1974), 437-473.

[Ja] K. Jacobs, Lipschitz functions and the prevalence of strictly ergodicity for continuous-time flows, Lecture Notes in Math., vol. 160, Springer-Verlag, 1970.

[Je] R. I. Jewett, The prevalence of uniquely ergodic systems, J. Math. Mech. 19 (1970), 717-729.

[K] W. Krieger, On unique ergodicity, Proceedings of the Sixth Berkeley Symposium on Mathematical Statistics and Probability 1970, pp. 327-346.

INSTITUTE OF MATHEMATICS, HEBREW UNIVERSITY, JERUSALEM, ISRAEL 